\title{
Acute Pulmonary Edema in Patients with Cushing's Syndrome
}

Mitra Niafar ${ }^{1}$, Mehrnoush Toufan ${ }^{2}$, Nooshin Milanchian ${ }^{3}$, Farhad Niafar², Kavous Shahsavari Nia ${ }^{4 *}$

1. Department of Endocrinology, Bone research center, Tabriz University of Medical Sciences, Tabriz, Iran

2. Department of Cardiology, Tabriz University of Medical Sciences, Tabriz, Iran

3. Department of Endocrinology, Urmia University of Medical Sciences, Urmia, Iran

4. Road Traffic Injury Research Center, Tabriz University of Medical Sciences, Tabriz, Iran

\section{Introduction}

Dyspnea refers to difficulty in breathing, and short and shallow breaths. This sign is seen in numerous diseases due to pulmonary, cardiac, metabolic and neurological causes. Among cardiac causes, heart failure is considered the main cause of dyspnea (1).

Cardiac failure is a clinical syndrome associated with a set of symptoms (dyspnea, and fatigue) and signs (edema and rales). Common causes of cardiac failure include: myocardial infarction, ischemic heart disease, hypertension, valvular heart diseases, and cardiomyopathy (2). Among uncommon causes of heart failure, endocrine disorders such as Cushing's syndrome can be cited. Cushing's syndrome can present itself in less common forms such as dyspnea due to heart failure. Cushing's syndrome's cardiovascular complications usually occur due to hypertension, end organ damage such as left ventricular heart failure, diastolic and ischemic myocardial heart failure, which are rather seen in chronic cases of the disease and are often irreversible (3).

Transient heart failure in patients with Cushing's syndrome, due to adrenal adenoma, has been reported in a number of patients. In this case report, a patient is introduced who presented to emergency department with severe dyspnea (FC III), and was ultimately diagnosed with Cushing's syndrome after work up. Three months after treatment of Cushing's syndrome, dramatic improvement was observed in this patient's cardiac function.

\section{The case}

The patient is a 35-year-old male, with a history of alcohol use, complaining of edema of the limbs and dyspnea in routine activities (FC III) within the previous 20 days. Patient's problems began nearly 2 years ago, in the form of gradual weight gain $(20 \mathrm{~kg})$ and elevated

\section{Corresponding author:}

Kavous Shahsavari Nia

Road Traffic Injury Research Center, Tabriz University of Medical Sciences, Tabriz, Iran

Receive date: 2014-06-20| Accept date: 2014-07-15| Publish date: 2014-07-27

DOI: 10.7575/aiac.abcmed.15.03.01.13 
blood pressure. In clinical examination, a Cushingoid (moon face) and plethoric facial appearance, with a buffalo hump were observed in the patient. In cardiac examination, there was a tangible heave in left ventricle and reduced $\mathrm{S} 1$ and $\mathrm{S} 2$, but audible $\mathrm{S} 3$ in auscultation. In auscultation of the lungs, fine crackles could be heard at caudal end of $1 / 3$ of lower lungs. In examination, abdominal obesity, and purple striae with more than $1 \mathrm{~cm}$ diameter were observed. Pitting edema was observed in lower extremities. Neurologic examination was normal other than slightly reduced tendon reflexes. Vital signs were as follows:

$B W=111 \mathrm{~kg} \quad \mathrm{BP}=110 / 70 \mathrm{mmHg} \quad \mathrm{BT}=37^{\circ} \mathrm{C}$ $\mathrm{RR}=24 / \mathrm{min} \quad \mathrm{HR}=110 / \mathrm{min}$
Given clinical results and the probability of Cushing's syndrome, 8 AM serum cortisol was requested for the patient and was reported as $16.33 \mu \mathrm{g} / \mathrm{dl}$. After the test, $1 \mathrm{mg}$ of overnight dexamethasone was administered and 8 AM serum cortisol was measured and reported as $21 \mathrm{\mu g} / \mathrm{dl}$. According to 24-hour UFC of three times the normal value, Cushing's syndrome was confirmed. Based on the results obtained, to investigate etiology of the syndrome, ACTH was requested, and considering the amount of $2.37 \mathrm{pg} / \mathrm{ml}$, adrenal Cushing was proposed.

Patient underwent hematological and biochemical tests whose results are presented in table 1.

\begin{tabular}{llllll}
\hline WBC & 700 & INR & 1 & LDL & 74 \\
\hline HB & 11.5 & PT & $12.5 \mathrm{sec}$ & HDL & 54 \\
\hline HCT & 34.2 & PTT & $30 \mathrm{sec}$ & Urea & 28 \\
\hline MCV & 92 & FBS & 104 & Cr & 1.5 \\
\hline MCHC & 31 & TG & 145 & Na & 148 \\
\hline PLT & 173000 & Chol & 165 & K & 4.6 \\
\hline
\end{tabular}

Table 1: Patient's hematological and biochemical tests

To assess etiology of Cushing's syndrome, spiral abdominal CT scan was requested, and a hypo-dense mass of $33 \times 29 \mathrm{~mm}$ dimensions was reported in the left adrenal gland. Cardiomegaly and blood congestion of the lungs were observed in patient's chest x-ray. Cardiac consultation and echocardiograph were performed on the patient, whose results are as follows:

EF $=30 \%$, mild Pericardial effusion, mild LVH, mild MR
Reduced LV and RV Systolic Function, Diastolic LV grade I dysfunction

Spirometry was performed to investigate pulmonary causes of dyspnea, with the following results:

FVC $=3.07 \rightarrow 65 \%$

FEV $1=2.69 \rightarrow 69 \%$

$\frac{F E V_{1}}{F V C}=87.62 \quad \mathrm{PEF}=\mathbf{7 . 7 6} \rightarrow \mathbf{8 4 \%}$

Impression: Moderate reduced in FVC, Lung volume study recommended 
Given the above results, diuretics and ACE inhibitor were started for the patient. Moreover, adrenalectomy was also performed on the patient. Three months after surgery, cardiac EF showed a significant improvement in echocardiography.

\section{Discussion}

Cushing's syndrome encompasses a set of clinical presentations, resulting from chronic exposure or persistent high blood glucocorticoids for any reason. The disease can be ACTH-dependent (such as: corticotrophic pituitary adenoma, or ectopic cases of ACTH tumor-maker) or ACTH-independent, such as: adrenal adenoma or iatrogenic causes, such as: exogenous use of glucocorticoids.

Cushing is a rare disease with incidence rate of 1 to 2 cases in every 100,000 people annually. Cushing's syndrome mostly appears as a set of non-specific signs and symptoms including obesity, diabetes, diastolic blood pressure, hirsutism, and depression. Therefore, clinical examination should be considered in suspected cases. Specific signs of Cushing's syndrome include fragile skin, frequent skin bruising, purple skin striae, and signs of proximal myopathy. Additionally, cardiovascular signs of Cushing's syndrome include: hypertension, hypokalemia, and atherosclerosis. To diagnose Cushing's syndrome, after ruling out exogenous causes (medication history), screening tests are performed, including 24-hour urine free cortisol test and overnight $1 \mathrm{mg}$ dexamethasone test. With positive screening test results, further diagnostic tests are used, including low dose dexamethasone test, and if confirmed, etiologic investigation is carried out by measuring plasma ACTH (3).

Below, a few studies on Cushing's syndrome in patients with heart problems are reviewed, in which, after treatment of underlying causes, normal cardiac function was resumed.
Only a few studies have been conducted with regards to cardiac function in Cushing's syndrome. However, in a study by De Leo et al., cardiac function was evaluated in patients with untreated Cushing's syndrome, and it was suggested that impaired LV filling could present following abnormal left ventricular geometry and increased LV mass; this also accompanies a slight increase in wall thickness and diastolic dysfunction in left ventricle. However, ventricular $E F$ and systolic function remain intact.

Recently, two-dimensional speckle tracking strain imaging technique has shown a significant improvement in LV structural and function abnormalities in Cushing's syndrome following serum cortisol level correction. Following treatment, a substantial decrease in LV mass and wall thickness in combination with normalized diastolic left ventricular function in patients with Cushing's syndrome were observed. Recovery in longitudinal and circumferential shortening and delayed relaxation in LV hypertrophy were also observed. After cortisol normalization, no significant blood pressure alteration was observed throughout follow-up suggesting the fact that changes in myocardial properties are independent from blood pressure (4).

Petramala et al. reported Cushing's syndrome in a 28-year-old woman presenting with dyspnea, orthopnea, nocturnal dyspnea, and generalized edema and following necessary work up, adrenal adenoma was reported. Recovery was observed in cardiac symptoms, following laparoscopic adrenalectomy [5]. Yong et al. reported Cushing's syndrome in a 28year-old woman, complaining of progressive dyspnea, orthopnea, hemoptysis, and inflammation of both lower limbs that had begun three weeks earlier. Following necessary work up, it was found that cardiac failure was caused by dilated cardiomyopathy due to right adrenal adenoma, and patient's problems 
gradually resolved after adrenalectomy (6) Johnson also introduced a 58-year-old woman, complaining about nocturnal dyspnea attack and bilateral ankle swelling. After necessary examinations, dilated cardiomyopathy was diagnosed, and bilateral adrenalectomy was performed in the context of Cushing's syndrome with ACTH-dependent unknown etiology, and patient's symptoms improved after treatment (7). Shibusawa introduced Cushing's syndrome due to adrenal adenoma in a 67-year-old woman that was admitted for secondary congestive heart failure due to dilated cardiomyopathy. Patient's cardiomyopathy reversed following necessary treatments (8). The patient with dyspnea reported by Pereira et al. had Cushing's syndrome, which had caused LVH and diastolic dysfunction, as well as left ventricular systolic dysfunction. Following necessary Cushing's syndrome treatment, cardiac changes returned to normal levels (9).

In the present study case, relative reduction was observed in symptoms after onset of cardiac failure treatment. Then, according to patient's clinical condition, and simultaneous Cushing's syndrome and cardiac failure, possibility of Cushing's syndrome was proposed for this failure. Following necessary work up, the patient underwent left adrenalectomy, in which benign adrenal mass (adenoma) was reported. After surgery, because of the suppressed contralateral adrenal, patient temporarily received $7.5 \mathrm{mg}$ daily dose of prednisolone. Three months later, echocardiography was performed and results confirmed improvement in cardiac failure.

\section{Conclusion}

Given all the above, the etiology should be sought in patients complaining about dyspnea. In the case of cardiac etiology, as well as investigating common causes, rare causes of cardiac failure, including endocrine causes, such as Cushing's syndrome, should also be considered. Once again, it should be reminded that congestive cardiac symptoms, like dyspnea and edema of limbs may be the first clinical presentation of Cushing's syndrome reported in every case. In the present study patient, after surgical or medicinal treatment of the syndrome, all cardiac symptoms were fully recovered.

\section{References}

1. Marx, J.A., et al., Rosen's emergency medicine: concepts and clinical practice. 2014.

2. Tintinalli, J., et al., Tintinalli's Emergency Medicine: A Comprehensive Study Guide, Seventh Edition (Book and DVD). 7th ed. 2010: Mcgraw-hill.

3. Melmed, S., et al., Williams Textbook of Endocrinology: Expert Consult. 12th ed. 2011: Elsevier Health Sciences.

4. De Leo, M., et al., Cardiovascular disease in Cushing's syndrome: heart versus vasculature. Neuroendocrinology, 2010. 92(Suppl. 1): p. 50-54.

5. Petramala, L., et al., Cushing's syndrome patient who exhibited congestive heart failure. Journal of endocrinological investigation, 2007. 30(6): p. 525.

6. Yong, T.Y. and J.Y. Li, Reversible dilated cardiomyopathy in a patient with Cushing's syndrome. Congestive Heart Failure, 2010. 16(2): p. 77-79.

7. Johnston, P.C., et al., An Unusual Cause of Reversible Cardiomyopathy. The Ulster medical journal, 2012. 81(3): p. 134.

8. Shibusawa, N., et al., Dilated Cardiomyopathy as a Presenting Feature of Cushing's Syndrome. Internal medicine (Tokyo, Japan), 2012. 52(10): p. 1067-1071.

9. Pereira, A.M., et al., Cardiac dysfunction is reversed upon successful treatment of Cushing's syndrome. European Journal of Endocrinology, 2010. 162(2): p. 331-340. 